\title{
Digital brain: Model-based framework for dependable electroencephalogram sensing and actuation in internet of things system
}

\author{
R. J. Kavitha ${ }^{1}$, K. K. Saravanan ${ }^{2}$ \\ ${ }^{1}$ Department of Electronics and Communication Engineering, Anna University, University College of Engineering, \\ Panruti, India \\ ${ }^{2}$ Department of Electrical and Electronics Engineering, Anna University, University College of Engineering, \\ Thirukkuvalai, India
}

\begin{abstract}
Article Info
Article history:

Received Apr 28, 2021

Revised May 27, 2021

Accepted Jun 28, 2021

\section{Keywords:}

Brain computer interface

Electroencephalogram

Human brain

Internet of things

Microcontroller

Virtual brain

ABSTRACT

Real-time brain internet of thing (IoT) frameworks are expensive. But, creating a cheaper framework has been quickened incredibly by the superior investigation that's being done on virtual brain. The passing of an imperative individual on a mystery mission is considered delicate data and must be taken care of with as much security as conceivable. By guaranteeing this discreteness, the time taken for the message of their passing to reach the pertinent specialist is expanded to up to a few days. The time taken to provide the message is as well. These days, the advancements in equipment expanding the capacities of the virtual brain and of the wearable brain IoT sensors have made the advancement of various unused program systems conceivable for engineers to make valuable applications that combine the human brain with IoT. Different tactile pathways are too empowered for communications of the human brain with bigger measured data. The fundamental point of this extend is to transfer secret records onto the clouds safely.
\end{abstract}

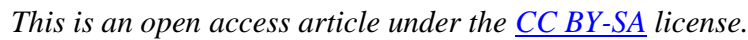

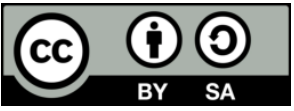

\section{Corresponding Author:}

K. K. Saravanan

Department of Electrical and Electronics Engineering, University College of Engineering Thirukkuvalai, Nagapattinam-Dist, Nearest Railway Station-Thiruvarur, Tamil Nadu, India E-mail: saravanan.santi@gmail.com

\section{INTRODUCTION}

Over a long time, all master and mass-showcase utilization circumstances counting sharp structure extending, exhibit the developing number of internet of things (IoT) things and its diverse organizations. A great understanding into how the brain of a human being works is inferred from a total picture of the structure of the brain. This information can be utilized to find unused drugs and strategies of treatment for a huge cluster of brain clutters [1]. The collection of an expansive volume of pictures of the brain tissues within the cellular and the sub cellular level has been made possible by the headways within the intaglio brain imaging strategies. These incorporate amplified investigation of the proteome (outline) and clarity [2]. In spite of the fact that this appears advantageous, these high-resolution images posture another challenge; to productively prepare and analyses them. The figures discharged final on livelihoods and of sent dependencies, appears an estimation of the IoT courses of action within the genuine scale operational circumstances. Additionally, pilot and commerce organizations are proposing the significance of utilizing the IoT standards in advanced utilization circumstances. The concept of brain IoT rotates around the complex 
circumstances wherein the actuation and control of a framework are reinforced by the populaces of the IoT containers [3].

The components that are utilized in this extend are an electroencephalogram (EEG) sensor, the AVR microcontroller, the IoT modem and the widespread non concurrent collector transmitter (UART). Electroencephalogram (EEG) is a sensor that's utilized to sense the electric beats of the human brain, independent of the reality that the brain is dynamic or not An AVR micro controller is utilized for utilizing the quick sign dealing with movement that's display in an embedded system. It as of now has all the highlights and is built onto a single chip. Brain computer interface (BCI) is the affinity of the human brain and the computer. UART is utilized for transmitting and collecting the information and it's being related with the IoT [4]. A modest bunch are arranged for a tall throughput and a long neuron examination at the phone degree, which is the premise for the understanding of the cerebral circuitry and for separating the significant and harmful psyches. A tall throughput and a moo dormancy request of intellect data will require a quick database and programming interface that is acceptable to the colossal scope of the diagrammatic assessment [5]. Brain charts offer a shifted system for the representation of the auxiliary or serviceable topology in numerous categories. A large number of computer program instruments exist for analyzing the topology of brain frameworks utilizing a chart hypothesis [6]. They basically center on inquiring about the relationships of the anatomically protects brain locales. A few are built for a tall throughput and long-range neural examination at the cellular level, which is pivotal for comprehending the brain entries and for relating dynamic and ailing brains [7]. Tall throughput and moo idleness calculation of brain information will command tall speed databases and programming interfacing that are effectively consistent with an enormous scale chart examination [8].

Neihart and Harrison [9] have spoken about the neural chronicle frameworks that need gadgets considering bidirectional information move. These gadgets should be little and low power as they are placed near to the cerebrum. Utilizing a parasitic radio wire under $2 \mathrm{~mm}$ long, a power level was estimated to be $59.73 \mathrm{~dB}$ m away off of one meter [9]. Kevric and Subasi [10] explain about three popular signal processing techniques (empirical mode decomposition, discrete wavelet transforms, and wavelet packet decomposition) were analyzed for the decomposition of electroencephalography (EEG) signals in the BCI system for a classification task. Publicly available BCI competition III dataset IVa, a multichannel 2-class motor-imagery dataset, was used for this purpose. Multiscale Principal Component Analysis method was applied for the purpose of noise removal [10].

Caldwell et al. [11] analyzed the effects of posture on electroencephalographic (EEG) activity and psychomotor vigilance task (PVT) performance in 16 sleep-deprived volunteers. EEG data were collected while participants completed 10 min PVTs under two counterbalanced sitting/standing conditions during $28 \mathrm{~h}$ of continuous wakefulness. These results suggest that an upright posture increases EEG arousal and sustained attention, indicating that postural manipulations can be useful for counteracting fatigue in sleep-deprived individuals [11]. The effect of posture on spontaneous brain dynamics by recording scalp electrical activity in four orthostatic conditions (lying supine, inclined at $45^{\circ}$, sitting upright, and standing erect). It shows that upright versus supine posture increases widespread high-frequency oscillatory activity. The electroencephalographic findings highlight the importance of posture as a determinant in neuroimaging. It generalizing supine imaging results to ecological human cognition; therefore, cognitive neuroscientists would benefit from considering the influence of posture on brain dynamics [12]. Technique fundamentally could be a show to clarify the techniques or strategies utilized to arrange, make or arrange a extend. This segment clarifies approximately the item and gear that will be utilized for building up this undertaking further. The results ought to be analyzed to achieve the objective of this project [13].

Speier et al. [14] describes about to develop a method that can overcome these barriers by optimizing EEG electrode number and placement for P300 studies within a population of subjects. A Gibbs sampling method was developed to find the optimal electrode configuration given a set of P300 speller data. This method presented a fining optimal electrode montages across a user population. Reducing the number of channels may reduce costs, set-up time, signal bandwidth, and computation requirements for practical online P300 speller implementation [14]. Two main challenges are arising in the existing IoT scenarios. One is the need to interconnect various unique platforms and smart things in a single environment [15]. A wizard for an SSVEP-based BCI that automatically determines individual BCI parameters for each user and can be handled by inexperienced personnel has been tested with 61 healthy subjects. The presented study confirms that through careful user-specific choice of SSVEP BCI constants such as stimulation frequencies, classification thresholds, and segment lengths, high accuracies can be achieved by a broad population. All subjects achieved reliable control over the BCI system, reaching accuracies above 85\% [16]. All these products are affordable, portable, and easy to use despite the variations in make and versions. These features are unquestionably well-placed, given that one of the primary goals for their creation is to attract a new community of commercial users [17]. 
Since conventional EEG has been used in clinical and experimental settings for years, the transition toward commercial and engineering sides has not been without uncertainty. With this in mind, researchers in related areas have been working diligently to ensure that these ostensibly innovative features were not implemented at the cost of reliability and accuracy, by performing validation studies comparing the output of data derived from consumer-grade EEG devices to information generated from standard research-grade counterparts [18]. Ear-EEG-based objective hearing threshold estimation evaluated on normal hearing subjects. The objective EEG based technique of auditory steady-state response has been successfully used in the clinic to estimate hearing threshold levels (ASSR) [19]. Brain-PC interface (BCI) approach will be executed in this paper [20].

\section{METHOD}

The diagram of the extend is exhibited in Figure 1. The portions utilized in this errand are EEG, AVR Small scale controller, IoT modem, UART wire, and constrain supply is enabled. Tall speed flag handling is the essential utilize of this AVR scaled down controller [21]. The AVR Small scale controller is related with the EEG sensor and IoT modem. The transformer is utilized in this investigation since the $240 \mathrm{v}$ can be changed over into $5 \mathrm{v}$. The transformer is related to the AVR Smaller scale controller. Lean film transmitter (TFT) is shown over the miniaturized scale controller. It is utilized for the reason to show. We ought to look at approximately the experimentation of the errand [22]. At the point when the constrain supply gets $\mathrm{ON}$, it is required to hold the EEG sensor on the brow and contact is required with hands. At the point when it is held, it comes about as brain energetic, in case not at that point the intellect isn't energetic. So, in that TFT appear, it appears as brain dead. The virtual intellect is enlivening the advancement of sensible continuous cerebrum PC interface (BCI). Hardware prerequisites that increase the capacity of the virtual mind are recorded underneath. The system architecture for model-based framework for dependable EEG sensing and actuation in IoT system is as shown in Figure 1.

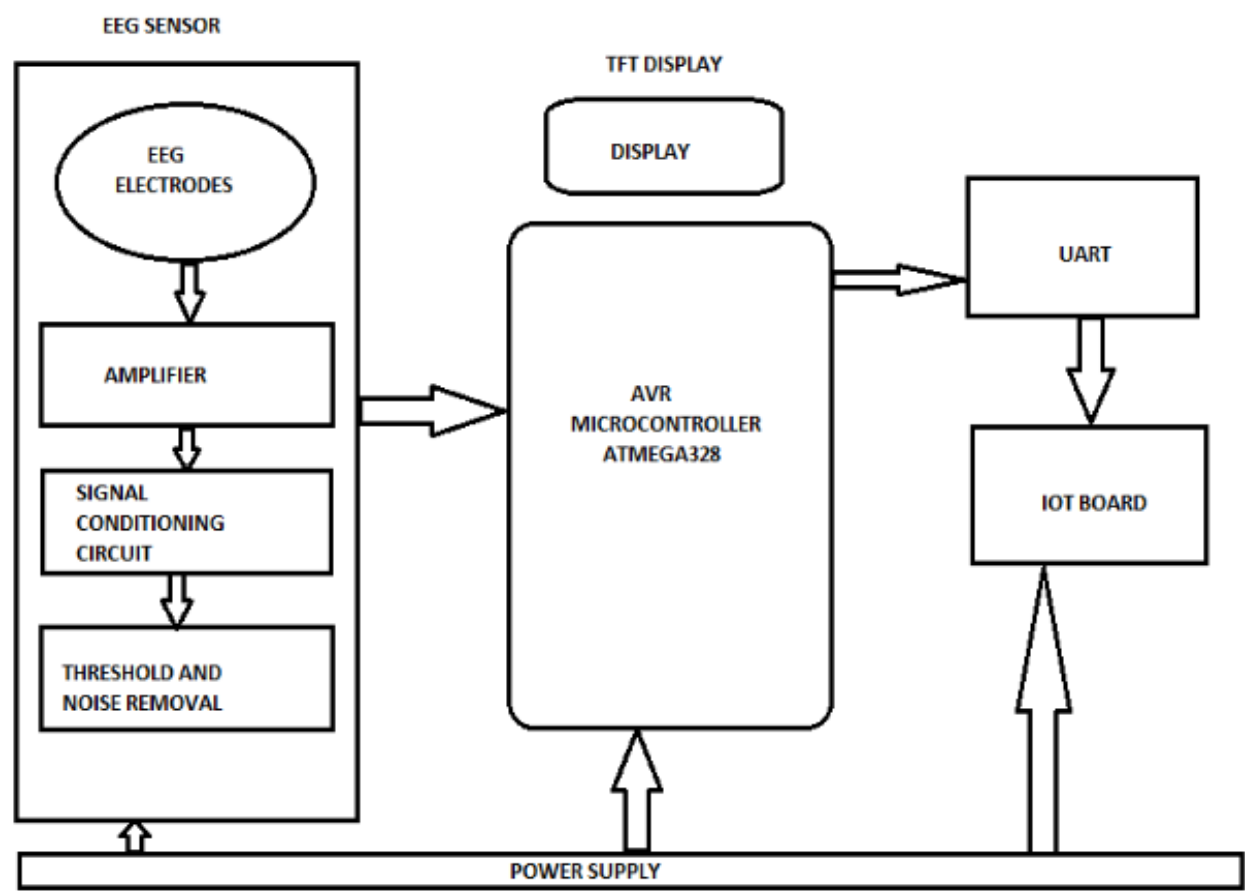

Figure 1. System architecture

\subsection{Virtual cerebrum module}

In this module, the components are getting facilitated. It comprises of parts like an electroencephalogram (EEG), AVR microcontroller, IoT modem, transformer UART board, in conclusion a TFT appear [23]. The AVR microcontroller is related with TFT appear and transformer. UART board is related with IoT modem and all these related with control supply. 


\subsection{Privacy data module}

In this module, the most key is to enter the username and secret word in arrange to reveal this screen. By tapping the choices like archive, mail id and phone number, the client can enlist the things and save them absent within the cloud. The information which stores in this module will be free from any protection issues.

\subsection{Privilege to access module}

In this module, a customized username and watchword may well be entered by the client. Fundamentally, this can be the module which is related with the electroencephalogram (EEG) contraption and PC. By entering the username and the watchword, the client can go into the taking after screen [24]. All clients ought to have an extraordinary username and watchword to ensure their security. The client can enter information which has to pass on to their family, companions or neighbor and can sort in their customized message, then again, there's a choice for them to spare their information.

\subsection{Remainder module}

At the point when the client embeds the security data on the cloud, the enlisted client will be informed by means of caution. The cloud is made such that it is profoundly secured and secure. On the off chance that a client or a client isn't lively, all the spared data and information may well be consequently sent to the enrolled mail id or mobile number.

\subsection{EEG sensor}

The fundamental work of the EEG sensor appeared in Figure 1 is to measure the electrical works out which is made by the synchronized activity of different neurons in volts which brings approximately an extraordinary objective in terms of time, which makes us to find the activity interior the imperative of different divisions of cortical locales indeed at sub-second timescales. EEG, the speediest imaging methods is available, and has high rate of test. Within the starting, when EEG sensor was established a various a long time back, it was plotted on paper. These days in current systems, it carefully appears the data as a nonstop movement of voltages [25].

\subsection{EEG signal}

EEG signal is decided in terms of recurrence and escalated. Flag concentrated is evaluated in microvolts $(\mu \mathrm{V})$.

\subsection{Microcontroller-Arduino}

Arduino microcontroller is one of the unimaginable single board PCs that have procured uncommon Arduino is open-source, which suggests that program of programming and improvement is free. Start fun is a not too bad source in Arduino hardware where US showcase is concerned. Arduino board can be utilized to create interface circuits to get it switches and distinctive sensors, and to control errands like motors and lights with less effort.

\subsection{Arduino Uno IDE}

Typically, a cross-stage application (for Windows, macOS, Linux) that's composed within the programming dialect Java and is utilized to compose and exchange ventures to Arduino practical sheets, and other shipper change sheets with the help of third-party vendors. The source code for the IDE is conveyed beneath the GNU Common Open Permit, variation. The Arduino IDE maintains the dialects $\mathrm{C}$ and $\mathrm{C}++$ utilizing a few special conditions with rules of code organizing. Arduino Uno is a microcontroller board based on the ATmega328P. It has 14 digital input/output pins (of which 6 can be used as PWM outputs), 6 analog inputs, a $16 \mathrm{MHz}$ ceramic resonator (CSTCE16M0V53-R0), a USB connection, a power jack, an ICSP header and a reset button. It contains everything needed to support the microcontroller; simply connect it to a computer with a USB cable or power it with an AC-to-DC adapter or battery to get started Arduino Uno microcontroller. Arduino Uno microcontroller is as shown in Figure 2.

\subsection{TFT display}

The TFT touch screen is an Arduino with practical vivid TFT appears with practical impression. The TFT driver has a capable driver IC with 8bit data and 4bit control interface. Able to draw message or do anything with the TFT library. It moreover has on board a smaller than expected SD card opening on the raise of the screen and store bitmap pictures to appear on the screen. The TFT library interfaces/connects with the controller of the screen through SPI when utilizing the TFT library. Whether or not it is modern improvement or ancient, each progression emanates a specific level of ruinous X-bar discharge. All that 
introductions can cause eye strain in case utilized for a long time with tall brightness. Different introductions such as TFT LCD, drove, Amoled does not utilize electron shaft thought. The Arduino IDE maintains the dialects $\mathrm{C}$ and $\mathrm{C}++$ utilizing a few interesting conditions with rules of code organizing.

\subsection{SP8266}

ESP8266 is a wide and independent Wi-Fi network resolution that can convey software applications, or through extra application processor uninstall all Wi-Fi networking abilities. ESP8266 when the device is mounted and as the only application of the application processor, the flash memory can be initiated directly from an exterior transfer. Built-in cache memory will support improve system performance and decrease memory necessities. Extra condition is when wireless internet access accepts the task of Wi-Fi adapter, you can improve it to any microcontroller-based design, and the connection is modest, just by SPI/SDIO crossing point or essential processor AHB bridge interface. Processing and packing size on ESP8266 influential part, it can be integrated via GPIO ports sensors and further applications exact apparatus to achieve the lowest primary in the growth and Process of at least occupy system resources. The ESP8266 extremely integrated chip, collected with antenna switch balun, power management converter, so with slight exterior circuitry, and holds front-end module, with the whole resolution intended to minimize the space occupied by PCB. The structure is prepared through ESP8266 established topmost features are: energy saving VoIP rapidly switch between the sleep/wake patterns, with low-power action adaptive radio bias, front-end signal processing functions, troubleshooting and radio structures exist features eliminate cellular/Bluetooth/DDR/LVDS/LCD interference. The pin diagram of ESP8266 is as shown in Figure 3.

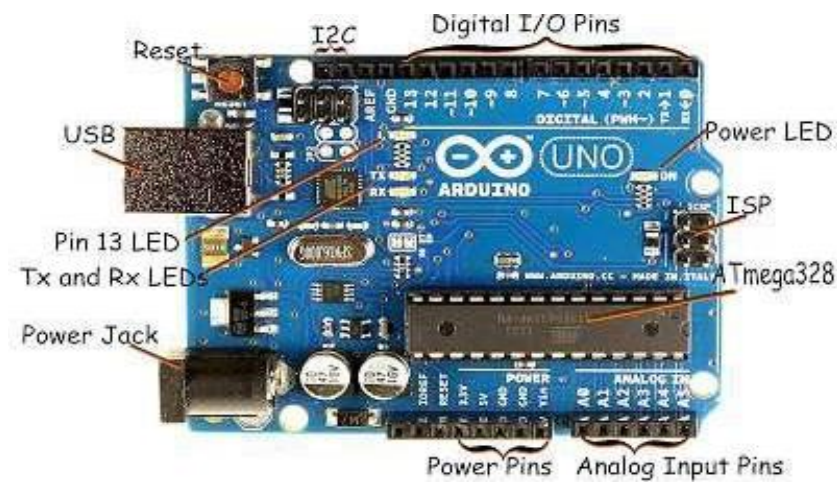

Figure 2. Arduino Uno

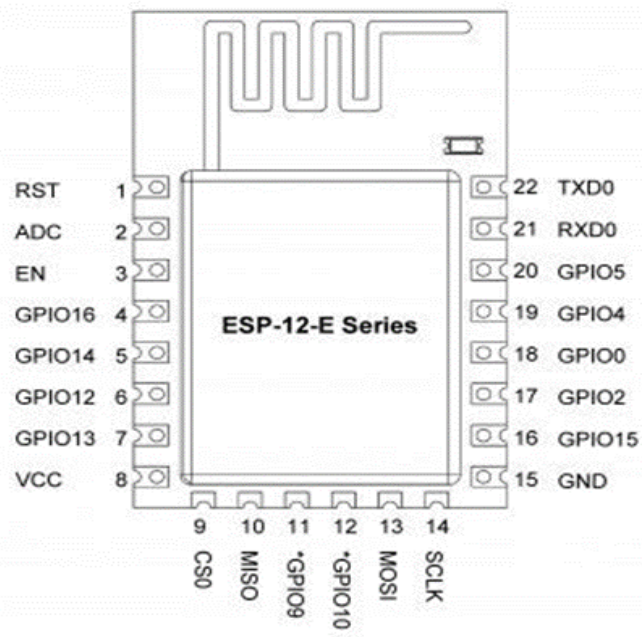

Figure 3. PIN diagram ESP8266 


\section{RESULTS AND DISCUSSION}

Here, at first the EEG headset with sensor is related with the chip Arduino board and it is related with the ESP8266 which is interconnected to the TFT Touchscreen [8]. To begin with the EEG headset sensors the EEG flag from the cerebrum with the terminals show within the headset and it checks whether it is lively or dead. Subsequent to identifying, given that the outcome comes is typical, it'll be appeared on the touch screen. Within the occasion that we require in Program application, able to check the circumstance with the intellect in Arduino IDE by utilizing the interface related with gear unit and the contraption is to be presented into the framework/PC in which Arduino programming has been presented. In devices menu, show within the Arduino application, press on the harbor which is empowered and after that we will direct see the circumstance with the cerebrum within the screen. Within the occasion that any alter or upgrade is seen, the status will be sent through SMS and mail id for express person in contact.

\subsection{Cloud Computing}

Cloud computing gathers taking care of and getting information and errands over the web rather than your PC's difficult drive. It is the blend of both equipment and programming. As usually clarified in this venture, also we will connect the contraption and web. Let's move on to the detailed explanation with respect to the program. Cloud computing, the act of utilizing a arrange of inaccessible laborers encouraged on the internet to store, direct, and prepare the data to neighborhood specialists or PCs. The login page of the computerized brain is as shown in Figure 4.

It comprises a client title and login. The item side of the proposed system is arranged by utilizing a hypertext preprocessing code. It may be a laborer side scripting dialect, moreover it is effortlessness of altering and doesn't include a parcel of complexity. It could be a backend dialect and within the proposed system, it needs a parcel of capacity, so hypertext preprocessing is able for that. As arranged for a one-on-one page, it very well may be made, by joining all the pages through the hypertext preprocessing dialect. It can moreover be utilized as taken a toll or freed from taken a toll depending upon the client.

Concurring to the benchmarks, able to enter the username and the secret word. With the objective that it joins in and get into the taking after page. By utilizing PHP code, these sites and pages can be arranged. Since it could be a cloud computing it comprises of username and secret word and it can't be open by Third-party. We will securely make up the location and location whereas coding. The over snap addresses the cerebrum status log nuances of the client. The brain status table is as shown in Figure 5.

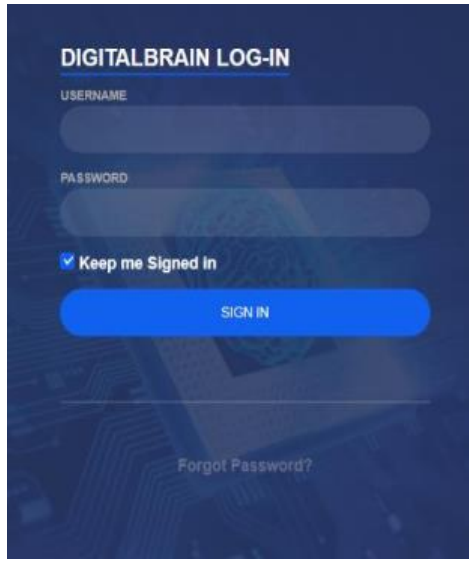

Figure 4. Login page
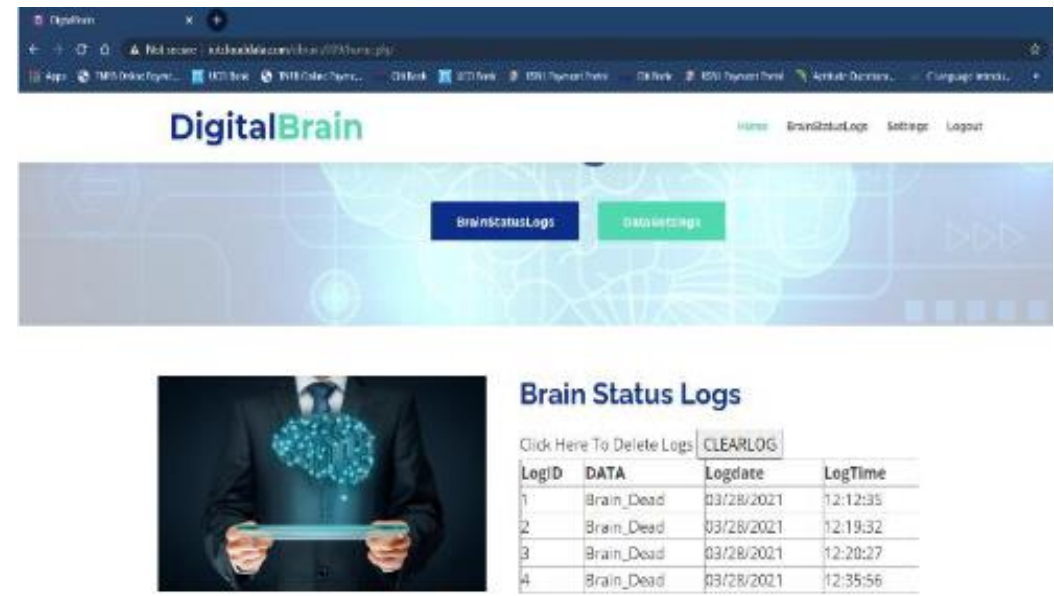

Brain Status Logs

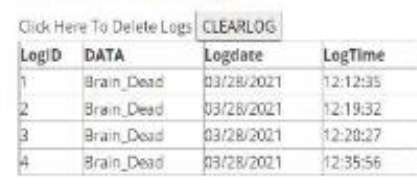

SETTINGS

Figure 5. Brain status table with date and time

At the point when the client is ordinary or when the client is dead, the data base accurately takes note the date and time of the clients. For occurrence, on the date of walk 28 , the client must have worn the contraption and appear the ordinary. Too, the other demonstrates the client's cerebrum is dead or the person is no more, the status log is get revived with the exact date and time. The status information set gets revived as needs are. 


\section{CONCLUSION}

In this proposed system, the result can absolutely ship off to the client's mail id or phone number. But within the situation that the client is lively, the client employments to sort the data which he/she has to be pass on to the best choice or particular person. As the result plainly shows it is the BCI. Wearable contraptions have made conceivable a couple of modern programming structures for engineers to utilize and make applications joining BCI and IoT. The file or information can be successfully gotten by a particular person instantly. On that note, this proposed system is more successful.

\section{REFERENCES}

[1] J. Kevric and A. Subasi, "The impact of MSPCA signal de-noising in real-time wireless brain computer interface system," Southeast Europe Journal of Soft Computing, vol. 4, no. 1, pp. 43-47, 2015, doi: 10.21533/scjournal.v4i1.90.

[2] M. Z. Baig, N. Aslam, H. P.H. Shum, and L. Zhang, "Differential evolution algorithm as a tool for optimal feature subset selection in motor imagery EEG," Expert Systems with Applications, vol. 90, pp. 184-195, 2017, doi: 10.1016/j.eswa.2017.07.033.

[3] V. P. Oikonomou, K. Georgiadis, G. Liaros, S. Nikolopoulos and I. Kompatsiaris, "A Comparison study on EEG signal processing techniques using motor imagery EEG data," 2017 IEEE 30th International Symposium on Computer-Based Medical Systems (CBMS), 2017, pp. 781-786, doi: 10.1109/CBMS.2017.113.

[4] D. Cheng, Y. Liu and L. Zhang, "Exploring motor imagery EEG patterns for stroke patients with deep neural networks," 2018 IEEE International Conference on Acoustics, Speech and Signal Processing (ICASSP), 2018, pp. 2561-2565, doi: 10.1109/ICASSP.2018.8461525.

[5] S. Kumar, R. Sharma, A. Sharma and T. Tsunoda, "Decimation filter with common spatial pattern and fishers discriminant analysis for motor imagery classification," 2016 International Joint Conference on Neural Networks (IJCNN), 2016, pp. 2090-2095, doi: 10.1109/IJCNN.2016.7727457.

[6] X. Guo, X. Wu, X. Gong and L. Zhang, "Envelope detection based on online ICA algorithm and its application to motor imagery classification," 2013 6th International IEEE/EMBS Conference on Neural Engineering (NER), 2013, pp. 1058-1061, doi: 10.1109/NER.2013.6696119.

[7] A. Nijholt, "The future of brain-computer interfacing (keynote paper)," 2016 5th International Conference on Informatics, Electronics and Vision (ICIEV), 2016, pp. 156-161, doi: 10.1109/ICIEV.2016.7759987.

[8] L. F. Nicolas-Alonso and J. Gomez-Gil, "Brain computer interfaces, a review," Sensors, vol. 12, no. 2, pp. 12111279, 2012, doi: 10.3390/s120201211.

[9] N. M. Neihart and R. R. Harrison, "Micropower circuits for bidirectional wireless telemetry in neural recording applications," in IEEE Transactions on Biomedical Engineering, vol. 52, no. 11, pp. 1950-1959, Nov. 2005, doi: 10.1109/TBME.2005.856247.

[10] J. Kevric and A. Subasi, "Comparison of signal decomposition methods in classification of EEG signals for motorimagery BCI system," Biomedical Signal Processing and Control, vol.31, pp. 398-406, 2017, doi: 10.1016/j.bspc.2016.09.007.

[11] J. A. Caldwell, B. Prazinko and J. L. Caldwell, "Body posture affects electroencephalographic activity and psychomotor vigilance task performance in sleep-deprived subjects," Clinical Neurophysiology, vol. 114, pp. 2331, 2003, doi: 10.1016/S1388-2457(02)00283-3.

[12] R. T. Thibault, M. Lifshitz, J. M. Jones, and A. Raz, "Posture alters human resting-state," Cortex, vol. 58, pp. 199205, 2014, doi: 10.1016/j.cortex.2014.06.014.

[13] K. Suefusa and T. Tanaka, "A comparison study of visually stimulated brain-computer and eye-tracking interfaces," Journal of Neural Engineering, vol. 14, no. 3, p. 036009, 2017, doi: 10.1088/1741-2552/aa6086.

[14] W. Speier, A. Deshpande and N. Pouratian, "A method for optimizing EEG electrode number and configuration for signal acquisition in P300 speller systems," Clinical Neurophysiology, vol. 126, pp. 1171-1177, 2015, doi: 10.1016/j.clinph.2014.09.021.

[15] Z. Tang, S. Sun, S. Zhang, Y. Chen, C. Li, and S. Che, "A brain-machine interface based on ERD/ERS for an upper-limb exoskeleton control," Sensors, vol. 16, no. 12, p. 2050, 2016, doi: 10.3390/s16122050.

[16] F. Gembler, P. Stawicki and I. Volosyak, "Autonomous parameter adjustment for SSVEP-based BCIs with a novel BCI wizard. Front," Neurosci, vol. 9, p. 474, 2015, doi: 10.3389/fnins.2015.00474.

[17] T. W. Picton, "The P300 wave of the human event-related potential," Journal of Clinical Neurophysiology, vol. 9, no. 4, pp. 456-479, 1992, doi: 10.1097/00004691-199210000-00002.

[18] P. Andersson, J. P. W. Pluim, J. C. W. Siero, S. Klein, M. A. Viergever, and N. F. Ramsey, "Real-time decoding of brain responses to visuospatial attention using 7T fMRI," PLOS ONE, vol. 6, no. 11, p. e27638, 2011, doi: 10.1371/journal.pone.0027638.

[19] L. Yao, Q. Z. Sheng and S. Dustdar, "Web-based management of the internet of things," in IEEE Internet Computing, vol. 19, no. 4, pp. 60-67, July-Aug. 2015, doi: 10.1109/MIC.2015.77.

[20] A. Vallabhaneni, T. Wang, and B. He, "Brain-computer interface," in Neural engineering, Springer, 2005, pp. 85121, doi: 10.1007/0-306-48610-5_3.

[21] A. Teles, M. Cagy, F. Silva, M. Endler, V. Bastos, and S. Teixeira, "Using brain-computer interface and internet of things to improve healthcare for wheelchair users," UBICOMM 2017 : The Eleventh International Conference on Mobile Ubiquitous Computing, Systems, Services and Technologies, 2017, pp. 92-94. 
[22] B. Jagadish, M. P. R. S. Kiran and P. Rajalakshmi, "A novel system architecture for brain controlled IoT enabled environments," 2017 IEEE 19th International Conference on e-Health Networking, Applications and Services (Healthcom), 2017, pp. 1-5, doi: 10.1109/HealthCom.2017.8210814.

[23] M. Bikson et al., "Rigor and reproducibility in research with transcranial electrical stimulation: An NIMHsponsored workshop," Brain Stimulation, vol. 11, no. 3, pp. 465-480, May 2018, doi: 10.1016/j.brs.2017.12.008.

[24] W. Legon, P. Bansal, R. Tyshynsky, L. Ai, and J. K. Mueller, "Transcranial focused ultrasound neuromodulation of the human primary motor cortex," Scientific Reports, vol. 8, no. 1, p. 10007, Dec 2018, doi: 10.1038/s41598-01828320-1.

[25] J. Kubanek, "Neuromodulation with transcranial focused ultrasound," Neurosurgical focus, vol. 44, no. 2, p. E14, Feb 2018, doi:10.3171/2017.11.FOCUS17621.

\section{BIOGRAPHIES OF AUTHORS}
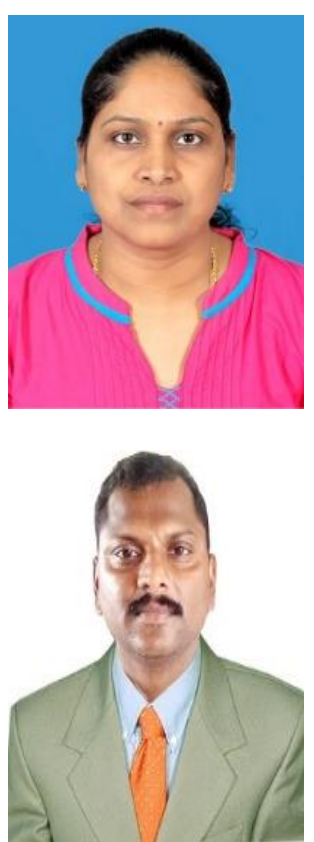

R. J. Kavitha is an Assistant Professor, Department of ECE in University College of Engineering, Panruti (A Constituent College of Anna University Chennai) since from Aug 2009. She graduated B.E. Electronics \& Communication Engineering from Government College of Engineering, Salem in 1998, and M.E. Optical Communication from A.C. College of Engineering \& Technology, Karaikudi in 2007 and Ph.D in Sep 2017 from Anna University. Her area of specialization is Wireless Sensor Networks, Digital Communication and Medical Electronics. She has 17 years of teaching experience. She has organized many events such as workshops, seminars, FDP, Symposiums. She has visited several countries like Qatar, Malaysia, and Singapore.

K. K. Saravanan is working as an Assistant Professor and Head (i/c), Department of Electrical and Electronics Engineering, University college of Engineering, Thirukkuvalai (A Constituent College of Anna University Chennai). He received the Bachelor degree in Engineering from Annamalai University, Chidambaram, India in 2002 Master of Engineering in 2005 from Sathyabama University, Chennai and Ph.D. in February 2018 from Anna University, Chennai, India. He has been in the teaching field for more than fifteen years. He has organized many events such as workshops, seminars, FDP, Symposiums. His areas of interests are Power Electronics Converters and its applications, Electrical machines, Materials in engineering, Renewable energy sources and others. 